\title{
Spectrum Leasing via Distributed Cooperation in Cognitive Radio
}

\author{
Igor Stanojev $^{1}$, Osvaldo Simeone ${ }^{1}$, Yeheskel Bar-Ness ${ }^{1}$ and Takki $\mathrm{Yu}^{2}$ \\ ${ }^{1}$ New Jersey Institute of Technology \\ Newark, New Jersey 07102-1982, USA \\ ${ }^{2}$ Samsung Electronics Co., Ltd. \\ Suwon, 442-600, Korea
}

\begin{abstract}
The concept of cognitive radio (or secondary spectrum access) is currently under investigation as a promising paradigm to achieve efficient use of the frequency resource. In this paper, we consider a decentralized cognitive radio model based on spectrum leasing, whereby a primary (licensed) user leases its bandwidth for a fraction of time to a network of independent secondary (unlicensed) terminals in exchange for cooperation. On one hand, the primary user decides whether to exploit (space-time coded) cooperation from the network of secondary terminals in order to maximize its own transmission rate. On the other hand, secondary terminals accept to cooperate with the primary only if compensated with a large enough fraction of time for their own transmission, towards the goal of maximizing their rate discounted by the overall cost of transmitted power. The considered model is studied in the framework of Stackelberg games, with the primary and the set of secondary users modelled as the (Stackelberg) game leader and the follower, respectively. Numerical results show that spectrum leasing based on trading secondary spectrum access for cooperation is a promising framework for cognitive radio.
\end{abstract}

\section{INTRODUCTION}

Cognitive radio is a debated new paradigm for efficient spectrum utilization that prescribes the coexistence on the same spectral resource of primary (licensed) and secondary (unlicensed) terminals. The most common approach to cognitive radio, often referred to as a commons or unlicensed model [1], requires secondary users to first sense the radio environment in search of spectrum holes (portions of the bandwidth where primary users are not active) and then opportunistically exploit the detected transmission opportunities. The main hindrance to the implementation of cognitive radio according to this principle is the inherent limitation in the ability of secondary nodes to properly sense the radio environment [2]. An alternative approach to secondary spectrum access, sometimes referred to as a property-rights model [1], prescribes primary users that are aware of the existence of secondary terminals on a given bandwidth, and willing to lease the spectrum for a fraction of time.

In this paper, we propose and study spectrum leasing in exchange for cooperation from the secondary users through distributed space-time coding [3]. In the considered model, a primary link (transmitter and receiver) shares a given

This work was supported in part by Samsung Electronics Co., Ltd. bandwidth with a secondary network consisting of multiple competing transmitter-receiver pairs (interference channel [4] [5]), as sketched in fig. 1. On one hand, the goal of the primary link is the maximization of its own achievable rate, by optimizing the amount of resources (fraction of time), if any, leased to the secondary network and the amount of required cooperation. On the other hand, different competing transmitters in the secondary network seek to increase their achievable rates towards the intended destinations under the constraints imposed by the resources leased by the primary and by the overall cost of transmission power (including the power spent for cooperation). Given the competitive nature of secondary transmitters, the outcome of their interaction can be conveniently described by a non-cooperative power control game [4] [5] and, more specifically, by the corresponding Nash Equilibrium (NE) ${ }^{1}[6]$.

An appropriate analytical framework to study the spectrum leasing scenario at hand is that of Stackelberg games [6]. In such a hierarchical game model, one agent (the competitive secondary network) acts subject to the strategy chosen by the other agent (primary link), which in turns seeks maximization of its own utility (here the achievable rate). Primary's strategy that yields the optimal solution and the corresponding power/cooperation response of the secondary network are jointly referred to as a Stackelberg equilibrium. The concept of a Stackelberg equilibrium can be further exploited to predefine a set of rules to be imposed on the players that would result in the most desirable interaction outcome. Related work can be found in [7], where the main focus is on promoting cooperation in ad hoc networks, and in [8], where the authors are primarily interested in the optimal design of an access point in a decentralized network.

\section{System Model}

In the following, we detail the proposed game-theoretic model of spectrum leasing and the main system parameters.

\section{A. Medium access control (MAC) layer}

We consider the system sketched in fig. 1, where a primary (licensed) transmitter PT communicates with the intended

\footnotetext{
${ }^{1}$ The Nash equilibrium is defined as the state whereby any unilateral deviation in player's strategy would not produce any gain [6].
} 
receiver PR within a slot whose duration is normalized to one. In the same bandwidth, a (unlicensed) secondary ad hoc network $\mathcal{S}$, composed of $K$ transmitters $\left\{\mathrm{ST}_{i}\right\}_{i=1}^{K}$ and $K$ receivers $\left\{\mathrm{SR}_{i}\right\}_{i=1}^{K}$, is active as well, seeking to exploit possible transmission opportunities. We assume one-to-one communication in $\mathcal{S}$, i.e., the data from the secondary terminal $\mathrm{ST}_{i}$ is intended for the secondary receiver $\mathrm{SR}_{i}$ (interference channel). Furthermore, without loss of generality, the secondary transmitters are sorted in descending order relative to the instantaneous channel power gain from PT.

The primary transmitter PT is assumed to be able to grant the use of the bandwidth to a subset $\mathcal{S}(k) \subseteq \mathcal{S}$ of $k$ secondary nodes in exchange for space-time coded cooperation so as to improve the quality of the communication link to its receiver PR. In particular, if the primary can benefit from cooperation (i.e., if it can achieve a larger rate than via direct transmission to the receiver PR), then it performs transmission as shown in fig. 1-(a). A fraction of the slot dedicated to its transmission towards the secondary set $\mathcal{S}(k)$ is of duration $1-\alpha(0 \leq$ $\alpha<1)$. Selection of the nodes in $\mathcal{S}(k)$ doesn't require further signalling but is obtained automatically via rate adaptation. Namely, only the terminals $\mathrm{ST}_{i}$ whose channels from PT are sufficiently good to support PT's rate are activated $\left(\mathrm{ST}_{1}\right.$ and $\mathrm{ST}_{2}$ in example depicted by fig. 1-(a)) .

The remaining time $\alpha$ is decomposed into two subslots according to a parameter $0 \leq \beta \leq 1$. In the first subslot of duration $\alpha(1-\beta)$, the $k$ active secondary nodes $\mathrm{ST}_{i} \in$ $\mathcal{S}(k)$ are allowed to transmit their own data (fig. 1-(b)), and the transmissions scheme amounts to an interference channel [4] [5]. The last subslot is of duration $\alpha \beta$ and is used for cooperation: the set $\mathcal{S}(k)$ of active $\mathrm{ST}_{i}$ form a distributed $k$ antenna array and cooperatively relay the primary codeword (decoded during the first subslot of duration $1-\alpha$ ) through distributed space-time coding towards PR [3] (fig. 1-(c)).

\section{B. Physical layer}

The channels between nodes are modeled as independent complex Gaussian random variables ${ }^{2}$, invariant within each slot (Rayleigh ergodic block-fading channels). We use the following notation: $h_{\mathrm{P}}$ denotes the complex channel gain between primary transmitter PT and primary receiver PR; $h_{\mathrm{PS}, i}$ the channel gain between $\mathrm{PT}$ and secondary transmitter $\mathrm{ST}_{i} ; h_{\mathrm{SP}, i}$ between $\mathrm{ST}_{i}$ and PR; $h_{\mathrm{S}, i j}$ between $\mathrm{ST}_{j}$ and $\mathrm{SR}_{i}$ for any $i, j=1, \ldots, K$. Without loss of generality, secondary nodes are sorted according to their channels from PT, i.e., $\left|h_{\mathrm{PS}, 1}\right|^{2} \geq\left|h_{\mathrm{PS}, 2}\right|^{2} \geq \ldots \geq\left|h_{\mathrm{PS}, K}\right|^{2}$, so that, according to the discussion above, we have $\mathcal{S}(k)=\{1,2, \ldots, k\}$. All the receivers have a perfect knowledge of the relevant channels, i.e., the $\mathrm{ST}_{i}$ and $\mathrm{PR}$ know the exact values of $h_{\mathrm{PS}, i}$ and $h_{\mathrm{SP}, i}$, respectively, for $i=1, \ldots, K$. Furthermore, the primary is assumed to be aware of all the instantaneous channel power gains in the system (i.e., $\left|h_{\mathrm{P}}\right|^{2},\left|h_{\mathrm{PS}, i}\right|^{2},\left|h_{\mathrm{SP}, i}\right|^{2}$ and $\left|h_{\mathrm{S}, i j}\right|^{2}$ ), while the knowledge of the channel power gains $\left|h_{\mathrm{S}, i j}\right|^{2}$ within

\footnotetext{
${ }^{2}$ Notice that the assumption of independent channels is generally used in the literature for the relay [3] and interference [4] channels.
}
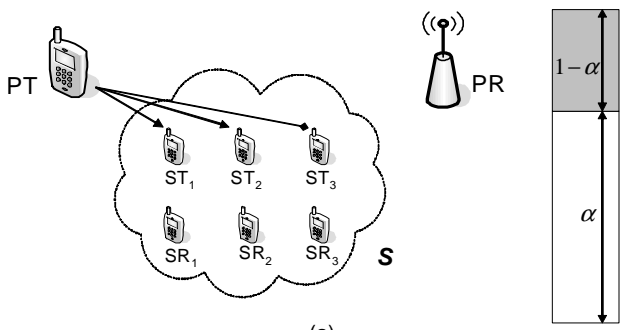

(a)
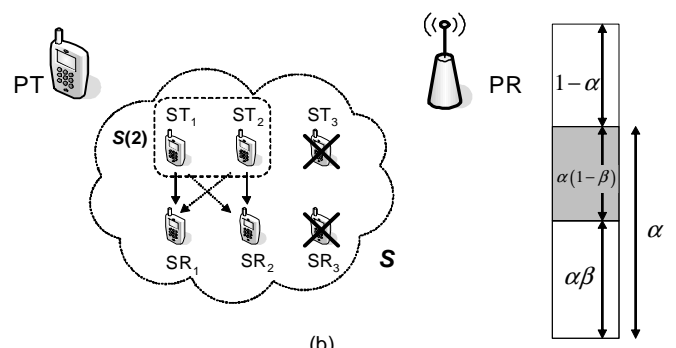

(b)

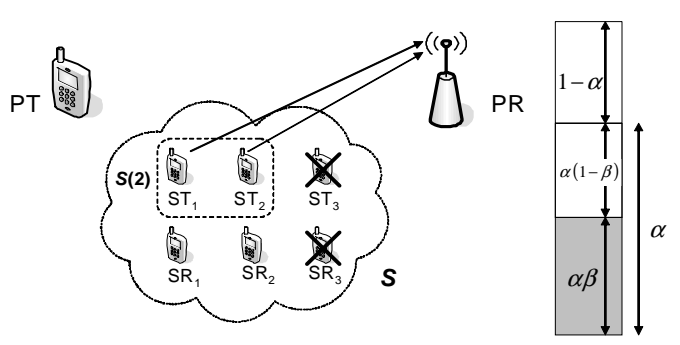

Fig. 1. Secondary spectrum access through cooperation-based spectrum leasing, for $K=3$ secondary transmitters and receivers: (a) primary transmission; (b) space-time coded cooperation; (c) secondary transmission.

the secondary network is required at the secondary terminals. Albeit ideal, the assumption of instantaneous Channel State Information $(\mathrm{CSI})^{3}$ is very common in the literature on gametheoretic applications to wireless networks (see, e.g., [4] [5]) and provides an interesting framework for analysis. A scenario with CSI knowledge limited only to the channel statistics is outside the scope of this work, and the related analysis and discussion can be found in [9], where randomized distributed space-time coding is considered. As for the synchronization issue on distributed space-time coding, we refer the reader to, e.g., [10].

The transmission power of the primary is denoted as $P_{\mathrm{P}}{ }^{4}$. On the other hand, secondary transmit powers $\mathbf{P}=\left[P_{1}, \ldots, P_{k}\right]^{T}, 0 \leq P_{i} \leq P_{\max }$ are obtained as the outcome $\hat{\mathbf{P}}$ (NE) of the power control game played between secondary nodes in the subslot of duration $\alpha(1-\beta)$ (fig. 1(b)), as detailed in Sec. III-B. During the relaying subslot (of duration $\alpha \beta$, fig. 1-(c)), the set of $k$ activated secondary nodes $\mathcal{S}(k)$ is constrained to use the same powers $\hat{\mathbf{P}}^{5}$ that are the

\footnotetext{
${ }^{3}$ This does not refer to the CSI at the receivers, as it can be easily facilitated using the training sequences.

${ }^{4}$ In this paper, power constrains are defined as average per transmitted symbol, as opposed to average over time.

${ }^{5}$ Possible malicious behavior of the secondary nodes in the relaying phase (i.e., using the power $P_{i}<\hat{P}_{i}$ or even refusing the cooperation, $P_{i}=\mathbf{0}$ ) is out of the scope of this paper.
} 
outcome of the power control game in the preceding subslot. Finally, the single-sided spectral density of the independent white Gaussian noise at the (both primary and secondary) receivers is $N_{0}$.

\section{GAME-THEORETIC ANALYSIS}

In this section, we describe and analyze the behavior of primary link and secondary network and discuss their interaction within a Stackelberg game framework.

\section{A. Primary link}

The primary link selects the slot allocation parameters $(\alpha, \beta)$ and the set of cooperating secondary nodes $\mathcal{S}(k)$ towards the aim of optimizing its transmission rate $R_{\mathrm{P}}(\alpha, \beta, k)$. As explained in Sec. II-A, we assume that the set $\mathcal{S}(k)$ is selected as $\mathcal{S}(k)=\left\{\mathrm{ST}_{i} \mid i=1, . ., k\right\}$, in order to simplify signalling ${ }^{6}$. Assuming decode-and-forward space-time coded cooperation from the set $\mathcal{S}(k)$ of $k$ active secondary users [3], the achievable rate reads

$R_{\mathrm{P}}(\alpha, \beta, k)=\left\{\begin{array}{l}\min \left\{(1-\alpha) R_{\mathrm{PS}}(k), \alpha \beta R_{\mathrm{SP}}(k, \beta)\right\}, \alpha> \\ R_{\mathrm{dir}}, \alpha=0\end{array}\right.$

The first line in (1) stands for the rate that is the outcome of cooperation (recall that $\alpha>0$ is the fraction of time slot dedicated for the secondary's activity). It is the minimum between two terms: $(i)$ the rate achievable in the first subslot (fig. 1-(a)) between the primary transmitter PT and the secondary transmitter $\mathrm{ST}_{k}$ (recall that, due to ordering, $\mathrm{ST}_{k}$ has the worst channel from PT within the set $\mathcal{S}(k)$ ), which is easily shown to be $(1-\alpha) R_{\mathrm{PS}}(k)$, where ${ }^{7}$

$$
R_{\mathrm{PS}}(k)=\log _{2}\left(1+\frac{\left|h_{\mathrm{PS}, k}\right|^{2} P_{\mathrm{P}}}{N_{0}}\right) ;
$$

(ii) the rate between the $k$ active secondary transmitters $\mathcal{S}(k)$ and the primary receiver PR via space-time coding (subslot highlighted in fig. 1-(c)), $\alpha \beta R_{\mathrm{SP}}(k, \beta)$, with

$$
R_{\mathrm{SP}}(k, \beta)=\log _{2}\left(1+\sum_{i=1}^{k} \frac{\left|h_{\mathrm{SP}, i}\right|^{2} \hat{P}_{i}(k, \beta)}{N_{0}}\right),
$$

where we have made explicit the dependence of the NE on the parameters $(k, \beta)$ selected by the primary link. Note that the rate (3) is obtained following the ideal information-theoretic assumption of orthogonal space-time coding able to harness the maximum degree of diversity from cooperation.

From (1), if the primary decides not to employ the cooperation, i.e., $\alpha=0$, then the primary rate is $R_{\mathrm{P}}(0, \beta, k)=R_{\mathrm{dir}}$, where

$$
R_{\text {dir }}=\log _{2}\left(1+\frac{\left|h_{\mathrm{P}}\right|^{2} P_{\mathrm{P}}}{N_{0}}\right)
$$

is the rate achievable on the direct link between primary transmitter PT and primary receiver PR.

\footnotetext{
${ }^{6}$ On the negative side, this choice requires every secondary terminal to attempt decoding.

${ }^{7}$ We assume random Gaussian codebooks.
}

The primary's optimization problem can now be summarized as:

$$
\begin{aligned}
& \max _{\alpha, \beta, k} R_{\mathrm{P}}(\alpha, \beta, k) \\
& \text { s.t. } k \leq K, 0 \leq \alpha, \beta \leq 1 .
\end{aligned}
$$

This problem can be interpreted as a Stackelberg game [6], whereby the primary is the Stackelberg leader, that optimizes its strategy $(\alpha, \beta, k)$ in order to maximize its revenue according to (5), aware that its decision will affect the strategy selected by the Stackelberg follower (the secondary set), i.e., the set of transmitting powers $\hat{P}_{i}(k, \beta)$.

\section{B. Secondary network}

Any active secondary terminal $\mathrm{ST}_{i}$ in the set $\mathcal{S}(k)$ attempts to maximize the rate towards its own receiver $\mathrm{SR}_{i}$ (discounted by the overall cost of transmission power), acting in a rational and selfish way and being aware of the parameters $(k, \beta)$ selected by the primary. In particular, each secondary transmitter $\mathrm{ST}_{i}$ chooses its transmitting power $P_{i}$ according to the NE $\hat{P}_{i}(k, \beta)$ (we will show that it exists and is unique) of the noncooperative power control game $\left\langle\mathcal{S}(k), \mathcal{P}(k), u_{i}\left(P_{i}, \mathbf{P}_{-i}\right)\right\rangle$. The set of allowed (power) strategies $\mathcal{P}(k)$ reads

$$
\mathcal{P}(k)=\left\{\mathbf{P}=\left(P_{1}, . ., P_{k}\right)^{T} \mid P_{i} \in\left[0, P_{\max }\right], i=1, . ., k\right\} .
$$

The utility function $u_{i}\left(P_{i}, \mathbf{P}_{-i}\right)$ of the $i$ th secondary node (player) is defined (similarly to, e.g., [11]) as the difference between the transmission rate $\alpha(1-\beta) R_{i}$ on the link between $\mathrm{ST}_{i}$ and $\mathrm{SR}_{i}$, where

$$
R_{i}\left(P_{i}, \mathbf{P}_{-i}\right)=\log _{2}\left(1+\frac{\left|h_{\mathrm{S}, i i}\right|^{2} P_{i}}{N_{0}+\sum_{\substack{j=1 \\ j \neq i}}^{k}\left|h_{\mathrm{S}, i j}\right|^{2} P_{j}}\right),
$$

and the energy cost $c \cdot \alpha P_{i}$ (recall that $\alpha$ is the fraction of time where the active secondary nodes are transmitting), with $c$ being the cost per unit transmission energy. Noticing from (7) that parameter $\alpha$ has no influence on the optimization process, we have

$$
u_{i}\left(P_{i}, \mathbf{P}_{-i}\right)=(1-\beta) R_{i}\left(P_{i}, \mathbf{P}_{-i}\right)-c \cdot P_{i},
$$

where $\mathbf{P}_{-i}$ is the vector that contains all the elements of $\mathbf{P}$ except the $i$ th (i.e., it denotes the set of other players' strategies). Notice that the utility of each node (8) depends on $k$ and parameter $\beta$, as well as on the power strategies of other activated users and the channel realizations. However, the only degree of freedom, i.e., the strategy available to the $i$ th secondary for the optimization of (8), is its transmission power $P_{i}$.

It is well known that a $\mathrm{NE}$ is a fixed point of the best responses of the nodes in $\mathcal{S}(k)$ [6]. Here, the best response of each user is obtained by setting the derivative of (8) with respect to $P_{i}$ to zero, i.e., $\partial u_{i}\left(P_{i}, \mathbf{P}_{-i}\right) /\left.\partial P_{i}\right|_{\mathbf{P}=\hat{\mathbf{P}}}=0$, for $i=1, \ldots, k$. It is possible to show that the NE $\hat{\mathbf{P}}$ is the solution of the following set of $k$ non-linear equations:

$$
\hat{P}_{i}=\left[\frac{1-\beta}{c}-\frac{N_{0}}{\left|h_{\mathrm{S}, i i}\right|^{2}}-\sum_{j=1, j \neq i}^{k} \frac{\left|h_{\mathrm{S}, i j}\right|^{2}}{\left|h_{\mathrm{S}, i i}\right|^{2}} \hat{P}_{j}\right]_{0}^{P_{\max }},
$$


where we used the following notation

$$
[x]_{x_{\mathrm{m}}}^{x_{\mathrm{M}}}=\left\{\begin{array}{l}
x, x_{\mathrm{m}} \leq x \leq x_{\mathrm{M}} \\
x_{\mathrm{m}}, x<x_{\mathrm{m}} \\
x_{\mathrm{M}}, x>x_{\mathrm{M}}
\end{array},\right.
$$

for any $x, x_{m}, x_{M} \in \mathcal{R}$. Therefore, the game has a unique NE if the system (9) has a unique solution. In particular, for given $\alpha$ and $k$, and $c=0$, the game $\left\langle\mathcal{S}(k), \mathcal{P}(k), u_{i}\left(P_{i}, \mathbf{P}_{-i}\right)\right\rangle$ has been discussed in the more general framework of wideband systems in [4] and [5], where it was shown that a NE exists and that it is unique if the matrix $\mathbf{H}$, defined as $[\mathbf{H}]_{i j}=\left|h_{\mathrm{S}, i j}\right|^{2}$ is strictly diagonally dominant, i.e.,

$$
\sum_{j=1, j \neq i}^{k} \frac{\left|h_{\mathrm{S}, i j}\right|^{2}}{\left|h_{\mathrm{S}, i i}\right|^{2}}<1
$$

The condition (11) for uniqueness of the NE is intuitive since it simply imposes an upper bound on the interference: in fact, with negligible interference equations (9) become uncoupled and the solution clearly exists and is unique. In the following, we assume that (11) holds. Finally, notice in (9) that if $\beta$ set by the primary is too large, the result can be the denial of cooperation by the secondary (by setting $\hat{P}_{i}=0$ ).

\section{Interaction between primary link and secondary network}

The interaction between the primary and the secondary network is modeled as a Stackelberg game [6], whereby the primary link is considered as the game authority, i.e., the Stackelberg leader. The leader optimizes its strategy $(\alpha, \beta, k)$ in order to maximize its revenue (1), knowing that its decision will affect the strategy selected by the Stackelberg follower (the secondary network), namely the set of transmitting powers $\hat{\mathbf{P}}$. The latter is in fact determined by the NE of the secondary power control game described in the previous section. Maximization of the revenue of the primary amounts to several trade-offs. For example, parameter $\beta$ has two conflicting effects on cooperation: while increasing $\beta$ entails more time for cooperation, it also renders cooperation from the secondary stations less likely since the cost induced by the transmitting power becomes dominant term in (8); furthermore, while a large value of $k$ may limit the overall rate by reducing the term $(1-\alpha) R_{\mathrm{PS}}(k)$ in (1), at the same time it enhances the term $\alpha \beta R_{\mathrm{SP}}(k, \beta)$ in (1) thanks to cooperation.

We now present some analytical insight into the considered system. Since the parameter $\beta$ appears only in the term $\alpha \beta R_{\mathrm{SP}}(k, \beta)$ of $(1)$, it can be optimized independently by solving the following optimization problem ${ }^{8}$ :

$$
\hat{\beta}=\arg \max _{\beta \in[0,1]} \beta R_{\mathrm{SP}}(k, \beta) \text {. }
$$

Moreover, for a given set $\mathcal{S}(k)$ and $\hat{\beta}$, the optimal fraction $\hat{\alpha}$ is given by making the two terms in the first line of (1) equal (so as to avoid performance bottlenecks), leading to:

$$
\hat{\alpha}=\frac{1}{1+\frac{\hat{\beta} R_{\mathrm{SP}}(k, \hat{\beta})}{R_{\mathrm{PS}}(k)}},
$$

\footnotetext{
${ }^{8}$ It can be proved that the optimization of (12) has a unique solution.
}

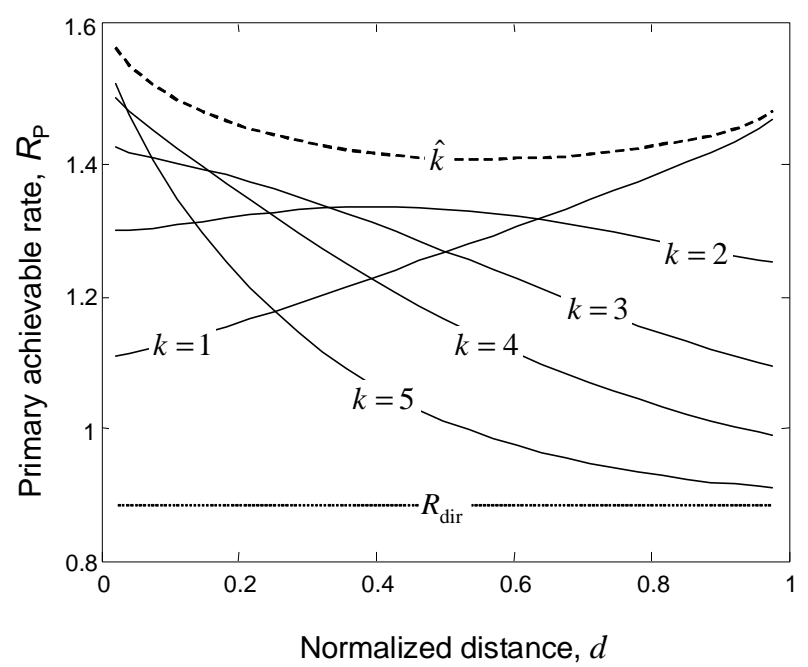

Fig. 2. Average primary rate $E\left[R_{\mathrm{P}}(\hat{\alpha}, \hat{\beta}, k)\right]$ (14) versus distance $d$, for different (fixed) $k$, and $k$ optimized for each fading realization ( $K=5$, $g_{\mathrm{S}}=10 \mathrm{~dB}$ and $\left.S N R=\mathbf{0} \mathrm{dB}\right)$.

and the optimized primary rate (1) reads

$$
R_{\mathrm{P}}(\hat{\alpha}, \hat{\beta}, k)=\frac{\hat{\beta} R_{\mathrm{SP}}(k, \hat{\beta}) R_{\mathrm{PS}}(k)}{R_{\mathrm{PS}}(k)+\hat{\beta} R_{\mathrm{SP}}(k, \hat{\beta})} .
$$

Recall from Sec. III-A that the primary decides to exploit the cooperation only if there exists some $k \leq K$ such that $R_{\mathrm{P}}(\hat{\alpha}, \hat{\beta}, k)>R_{\mathrm{dir}}$; otherwise it uses the direct link with achievable rate $R_{\text {dir }}$. Furthermore, we note that the optimization over the parameter $\beta$ (12) and over the number of users $k$ (as in (14)) requires numerical solving methods. The next section provides numerical results to corroborate these conclusions.

\section{NumericAl Results}

In this section, we consider a simple geometrical model where the set of secondary nodes is placed at a normalized distance $0<d<1$ from the primary transmitter PT and $1-d$ from the primary receiver $\mathrm{PR}^{9}$. Consequently, considering a path loss model, the average channel power gains read: $E\left[\left|h_{\mathrm{P}}\right|^{2}\right]=1, E\left[\left|h_{\mathrm{PS}, i}\right|^{2}\right]=1 / d^{\gamma}$, and $E\left[\left|h_{\mathrm{SP}, i}\right|^{2}\right]=1 /(1-$ d) $)^{\gamma}$, where $\gamma=2$ is the path loss coefficient. Moreover, we set $E\left[\left|h_{\mathrm{S}, i j}\right|^{2}\right]=1$ for $i \neq j,, P_{\mathrm{P}}=1, P_{\max }=6$ and $c=0.2^{10}$.

In order to get insight into the optimal behavior of the secondary network, and in particular on the optimal number of activated nodes $k$, fig. 2 shows the achievable rate (14) of the primary averaged over different (fading) channel realizations, $E\left[R_{\mathrm{P}}(\hat{\alpha}, \hat{\beta}, k)\right]$, versus the normalized distance $d$, with optimized parameters $\hat{\alpha}$ and $\hat{\beta}$ (recall (12) and (13)), different $k=1, \ldots, K, K=5, g_{\mathrm{S}} \triangleq E\left[\left|h_{\mathrm{S}, i i}\right|^{2}\right]=10 \mathrm{~dB}$ and

\footnotetext{
${ }^{9}$ While this collocation pattern can raise questions about the channel independence assumption, we emphasize that the model is stated in such a manner strictly for the tractability of the numerical examples.

${ }^{10}$ For the optimization over $\beta$ and $k$, the exhaustive search method is used. Furthermore, the results are valid for the system realization where the condition (11) holds.
} 


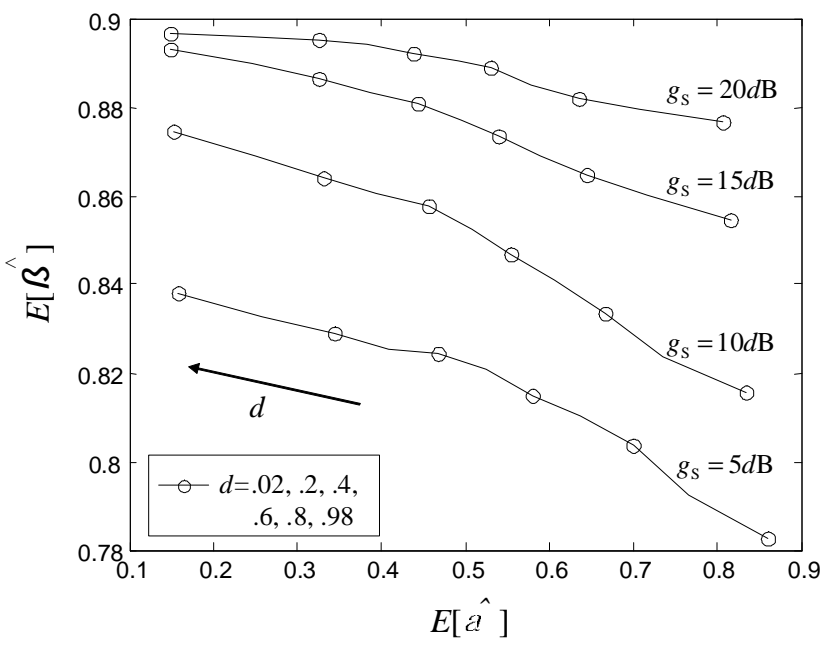

Fig. 3. Averaged optimum parameters $E[\hat{\alpha}]$ and $E[\hat{\beta}]$ versus distance $d$, for different number of secondary transmitters $K$ and $g_{\mathrm{S}}(S N R=\mathbf{0} \mathrm{dB})$.

$S N R \triangleq P_{\mathrm{P}} / N_{0}=0 \mathrm{~dB}$. The result of the optimization over $k$ is shown as well and the average rate with no cooperation $\left(R_{\text {dir }}, \alpha=0\right)$ is plotted as a reference. The figure reveals that the proposed cognitive scenario with cooperation (and properly chosen parameters) improves the (average) system performance. It also shows that larger gains from cooperation can be harnessed if the secondary nodes are sufficiently close to the primary transmitter (similar to [3]). Furthermore, it can be seen that for small distances it is better to activate (and thus cooperate with) a large number of secondary users given the large channel power gain from source to secondary network. Conversely, for large distances it is more convenient to cooperate only with the secondary users with the best instantaneous channel $\left|h_{\mathrm{PS}, i}\right|^{2}$ exploiting multiuser diversity. Further insight into the system performance, for optimized $(\hat{\alpha}, \hat{\beta}, \hat{k})$, is provided through the following numerical experiments.

Parameters $(\hat{\alpha}, \hat{\beta})$ that maximize the primary rate (1) are shown, averaged over fading, in fig. 3 for varying normalized distance $d$ and different values of $g_{\mathrm{s}}$, fixed $K=5$ and $S N R=$ $0 \mathrm{~dB}$. It can be seen from these curves that, as the distance $d$ increases, less time $\hat{\alpha}$ is devoted to the secondary network, but relatively more cooperation (reflected in $\hat{\beta}$ ) is required, as anticipated in Sec. III-C. Notice from fig. 3, however, that compared to $\hat{\alpha}$, the parameter $\hat{\beta}$ spans much smaller range. Moreover, the larger values of $g_{\mathrm{S}}$ induce larger $\hat{\beta}$, as the primary exploits the fact that the decreased interference makes secondary nodes more inclined to cooperate (recall (9)).

Finally, fig. 4 represents the mean rate of the secondary user (7), $R_{i}=\frac{1}{K} \alpha(1-\beta) \sum_{i=1}^{k} R_{i}$, averaged over fading, as a function of $d$, for different values of $g_{\mathrm{S}}$ and fixed $K=5$ and $S N R=0 \mathrm{~dB}$. It confirms that the secondary users benefit from larger $g_{\mathrm{S}}$. Furthermore, as the distance $d$ increases, the average rate of the secondary nodes becomes smaller, due to the decreased number of activated secondary transmitters (as seen in fig. 2).

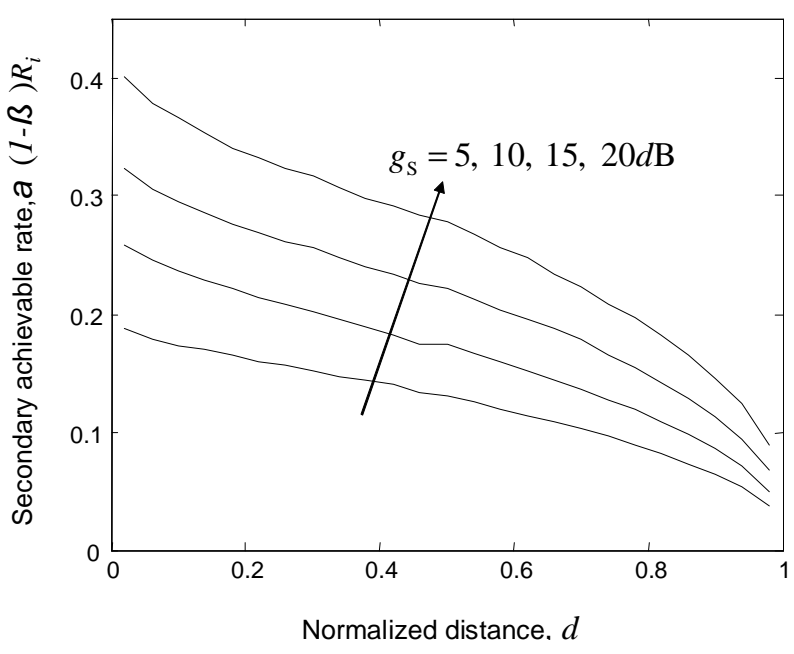

Fig. 4. Average secondary rate $E\left[R_{i}\right]$ (14) versus distance $d$, for different $g_{\mathrm{S}},(K=5$ and $S N R=\mathbf{0} \mathrm{dB})$.

\section{CONCLUDING REMARKS}

In this paper, we have investigated a secondary spectrum access scheme that hinges on cooperation-based spectrum leasing. Analysis has been carried out in the framework of Stackelberg games. Numerical results confirm that the considered model is a promising paradigm for cognitive radio networks.

\section{REFERENCES}

[1] J. O. Neel, Analysis and Design of Cognitive Radio Networks and Distributed Radio Resource Management Algorithms, Ph.D. dissertation, Virginia Polytechnic Institute, September 2006.

[2] A. Sahai, N. Hoven and R. Tandra, "Some fundamental limits on cognitive radio," in Proc. Allerton Conference on Communication, Control and Computing, 2004.

[3] J. N. Laneman and G. W. Wornell, "Distributed space-time coded protocols for exploiting cooperative diversity in wireless networks," IEEE Trans. Inform. Theory, vol. 49, no. 10, pp. 2415-2425, Oct. 2003.

[4] R. Etkin, A. Parekh and D. Tse, "Spectrum Sharing for Unlicensed Bands," in Proc. Allerton Conference on Communication, Control and Computing, 2005.

[5] G. Scutari, D. P. Palomar and S. Barbarossa, "Optimal Linear Precoding/Multiplexing for Wideband Multipoint-to-Multipoint Systems based on Game Theory-Part I: Nash Equilibria," to appear in IEEE Trans. on Signal Processing.

[6] M. J. Osborne and A. Rubenstein, A Course in Game Theory, MIT Press, 1994.

[7] O. Ileri, S. C. Mau and N. B. Mandayam, "Pricing for Enabling Forwarding in Self-Configuring Ad Hoc Networks," IEEE J. Select. Areas Commun., vol. 23, No. 1, pp. 151-162, Jan. 2005.

[8] I. Stanojev, O. Simeone and Y. Bar-Ness, "Optimal Design of a MultiAntenna Access Point with Decentralized Power Control Using Game Theory," in Proc. IEEE DySPAN 2007.

[9] O. Simeone, I. Stanojev, S. Savazzi, Y. Bar-Ness, U. Spagnolini and R. Pickholtz, "Spectrum Leasing Based on Distributed Space-Time Coded Cooperation with a Competitive Secondary Ad Hoc Network," submitted to IEEE J. Select. Areas Commun.

[10] X. Li, "Space-Time Coded Multi-Transmission Among Distributed Transmitters Without Perfect Synchronization," IEEE Signal Processing Letters, vol. 11, no. 12, pp. 948-951, Dec. 2004.

[11] J. Huang, R. A. Berry and M. L. Honig, "Distributed Interference Compensation for Wireless Networks," IEEE Journ. Selected Areas Commun., vol. 24, no. 5, pp. 1074-1084, May 2006. 